\title{
Eruptive Keratoacanthomas within a Red Ink Tattoo: A Case Report
}

\author{
Brayden Healey ${ }^{1}$, William Galbraith ${ }^{1}$, Yasser M. Sammour ${ }^{2}$, Diane Baird ${ }^{3}$ \\ ${ }^{1}$ Western University of Health Sciences College of Osteopathic Medicine of the Pacific-Northwest, Lebanon, USA \\ ${ }^{2}$ Cleveland Clinic, Cleveland, USA \\ ${ }^{3}$ Diane Baird Dermatology, Eugene, USA \\ Email: ^bhealey@westernu.edu
}

How to cite this paper: Healey, B., Galbraith, W., Sammour, Y.M. and Baird, D. (2018) Eruptive Keratoacanthomas within a Red Ink Tattoo: A Case Report. Case Reports in Clinical Medicine, 7, 470-475. https://doi.org/10.4236/crcm.2018.78041

Received: July 9, 2018

Accepted: August 10, 2018

Published: August 13, 2018

Copyright (c) 2018 by authors and Scientific Research Publishing Inc. This work is licensed under the Creative Commons Attribution International License (CC BY 4.0).

http://creativecommons.org/licenses/by/4.0/

\begin{abstract}
Background: There has been a longstanding debate regarding whether keratoacanthomas (KAs) are neoplastic or reactive/inflammatory lesions. Aim: The aim of this case report is to, within the aforementioned debate, offer support in favor of the potential reactive nature of keratoacanthomas. Case Presentation: A 64-year-old male presented with an eruption of 25 keratoacanthomas within a red ink tattoo. Eruptions were partially resolved over a matter of months, and completely resolved with treatment using oral Acitretin therapy. We believe this to be the third such reported case of eruptive KAs within only the red ink portions of a tattoo. Prior cases involved 2 and 8 KAs each. Discussion: Multiple studies suggest that KAs are neoplastic in nature and very distinct from cutaneous squamous cell carcinoma. Especially interesting is the finding that KAs have increased expression of apoptotic genes; this is particularly notable because of the tendency for these lesions to spontaneously involute. Skin tumorigenesis can occur in predisposed individuals after carcinogen exposure, thus red ink possesses theoretical potential as a carcinogen. KAs could be seen in this case as in fact a reactive neoplasm.
\end{abstract}

\section{Keywords}

Keratoacanthoma, Squamous Cell Carcinoma, Tattoo, Red Ink, Acitretin

\section{Introduction}

Keratoacanthomas (KAs) are squamous cell variant neoplastic lesions frequently encountered in clinical dermatology [1] [2]. Their exact etiology is yet to be clearly understood, though most recently the literature suggests they are a folli- 
cular variant of cutaneous squamous cell carcinoma (SCC). Nevertheless, KAs have been shown to react differently than most cutaneous neoplasms [3]. Clinically, they present distinctly as rapidly progressing erythematous papules and nodules that evolve to contain a hyperkeratotic, crateriform central crust. They often progress rapidly and have potential for spontaneous involution [4]. Despite this, KAs are often treated clinically as SCC and excised before spontaneous involution occurs. This is due to the clinical and histopathologic difficulty in distinguishing the two. Various exposures and risk factors have been associated with the development of KAs, though typically the lesions are spontaneous [3]. Multiple genetic syndromes have been described in which the eruption of multiple KAs can occur, however in non-syndromal patients eruptive lesions is rare. In certain case reports, various traumatic insults have been suggested to cause KAs to occur, potentiating a reactive or koebnerization-like tendency of KAs. The potential for tattoo ink to act as one such inciting insult has not been well described, despite the multiple other diseases known to be associated with tattoos. A total of 17 cases have been reported in which a KA has occurred within the red ink areas of cutaneous tattoos [5]. We report a case of eruptive KAs occurring entirely within red inked portions of a tattoo.

\section{Case Presentation}

A 64 year-old male presented to a dermatology clinic with multiple lesions that developed over the past 6 weeks with no prior treatment or evaluation. Sixteen months prior, the patient had obtained a solid black full sleeve tattoo with a 3-inch thick red circumferential band just above the elbow. The original red portion was inked with Lipstick Red ink by Eternal. This tattoo healed entirely as expected. Four months prior to presentation, the red band was re-inked with Dark Red ink, also by Eternal. Three days later, the patient began to notice a deep, burning sensation within the red band; this progressed to the development of painful, draining pustules and papules, which waxed and waned but progressed overall for the next 8 weeks until presentation. Upon presentation, he was found to have approximately 25 tender, well-circumscribed papules, some with hyperkeratotic, crusted centers, located entirely within the red-ink band (Figure 1). Two punch biopsies were obtained. Pathological evaluation showed results of invasive, well-differentiated squamous cell carcinoma in both samples. Appearing pathologically as SCC (Figure 2) and clinically as eruptive KAs, oral Acitretin $10 \mathrm{mg}$ daily was prescribed. The patient did not start the medication until his next office visit eight weeks later. Interestingly, at that follow up with no treatment as of yet, some improvement of lesions was noted on exam. Ten weeks later, he followed up with near complete resolution of the lesions after using Acitretin $10 \mathrm{mg}$ daily (Figure 3). Nearly one year later, the patient continues to have nearly full resolution and has developed any new lesions since the initial eruption. The patient gave full consent for the case as well as photos to be published. 


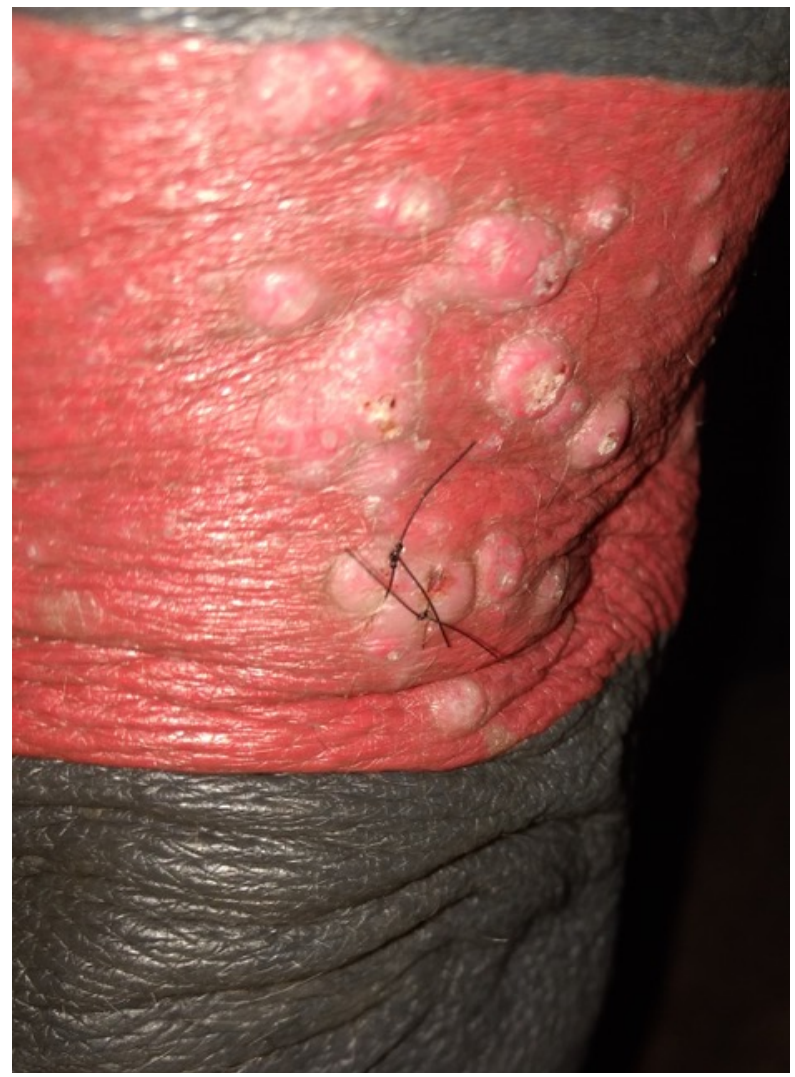

Figure 1. Seen entirely within the red portions of this sleeve tattoo are multiple crusted, crateriform papules clinically representative of keratoacanthomas. This image was taken at the time of biopsy.

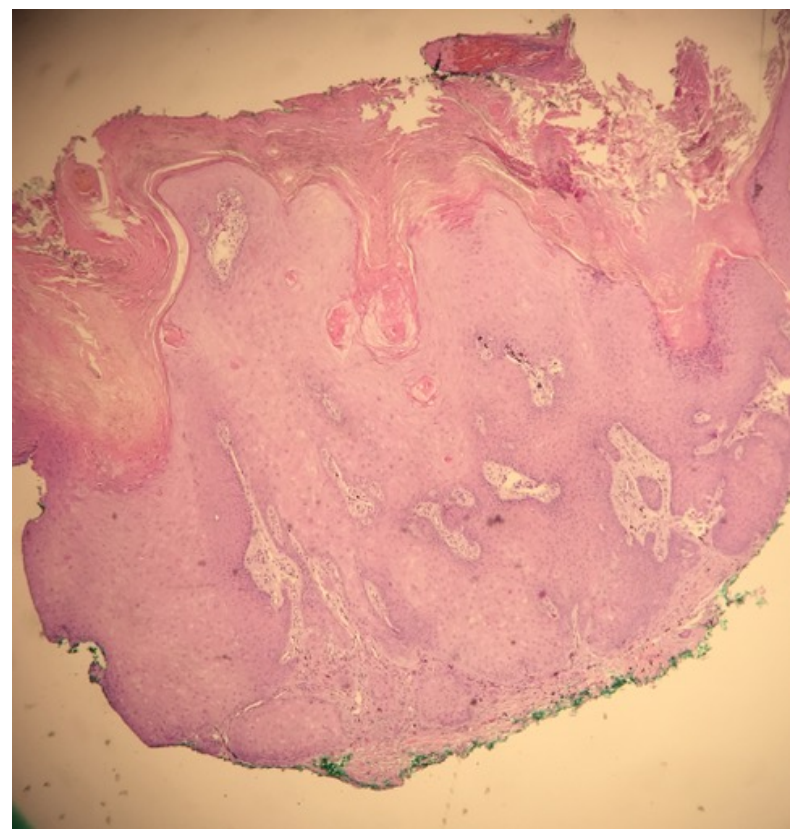

Figure 2. H \& E stained sections of the specimen show a punch biopsy of skin with acanthosis, hyperkeratosis and parakeratosis. A central, endophytic squamous proliferation is present with dyskeratosis and cytologic atypia. Associated tattoo pigment is present in the interstitium with solar elastosis and chronic inflammation. 


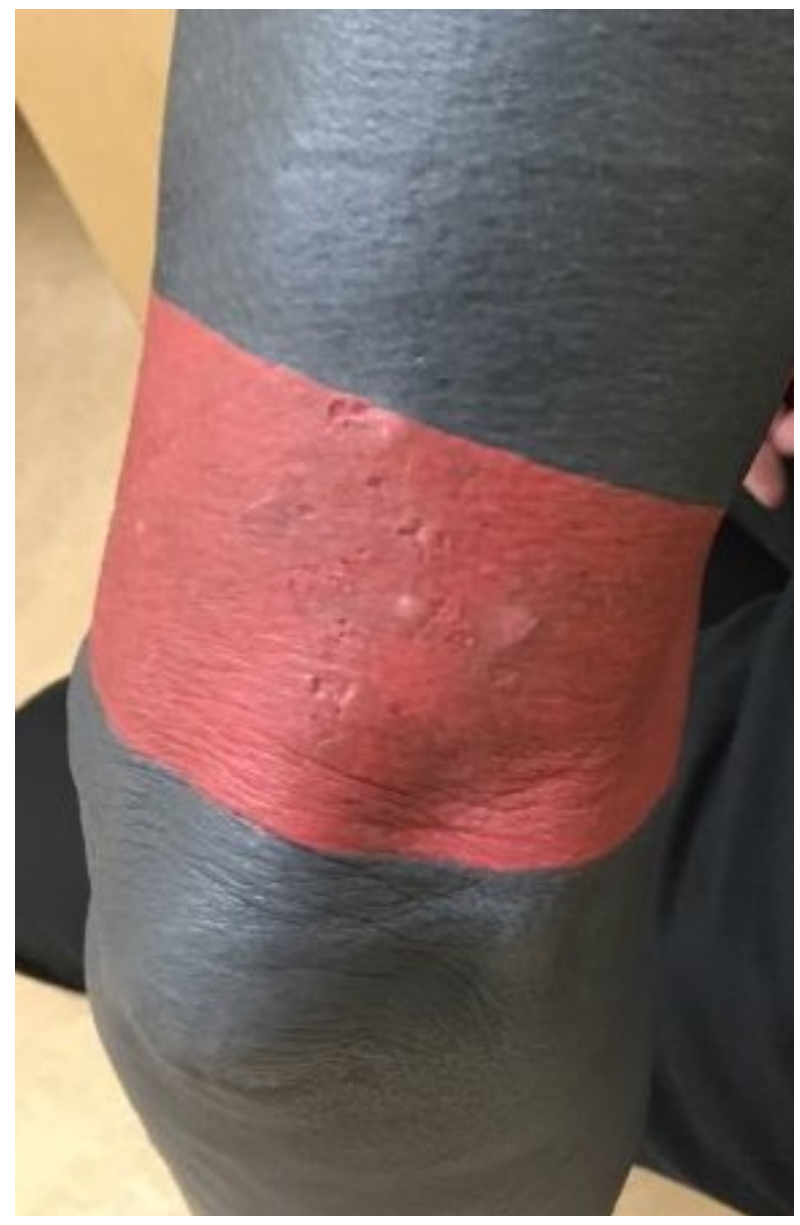

Figure 3. After ten weeks on Acitretin therapy, the lesions had fully regressed and all that remained in their place were a few small crateriform or papular scars without loss of tattoo pigment.

\section{Discussion}

KAs are most frequently recognized as a variant of follicular SCC [6]. They have a tendency to develop quickly and clinically present as solitary papules ranging from 0.5 to $2 \mathrm{~cm}$ in diameter. Maturity of the lesion leads to a pink-red nodule with a crateriform, hyperkeratotic center. Differentiation from SCC, amelanotic melanoma, and basal cell carcinoma is often not possible with physical examination alone. Growth typically continues for months and is often followed by involution over the same time span. Notwithstanding, many lesions are treated before spontaneous involution due to the difficulty in differentiation from other neoplasms [7].

Much debate has been had in classifying KAs as neoplastic or reactive. They have been found to occur in areas of trauma, surgery, chemical irritation, and UV exposure [3]. Most commonly, however, they are found to be isolated and apparently spontaneous. Rare cases of eruptive lesions are encountered mainly with genetic syndromes [6]. The appearance of eruptive KAs confined to a red ink tattoo is inconsistent with these known syndromes. 
The etiology of KAs remains unclear. In many instances, as in this case, pathologists do not differentiate KAs from SCC, or label KAs as subtypes of SCC. Recent studies have suggested that both molecularly and clinically, KAs are distinct from SCC and should be treated accordingly. A clinical systematic review argues strongly for the benign nature of the lesions, citing that of 445 cases studied over time, zero cases of metastasis were reported and no deaths occurred at the hand of the disease. To the contrary, 5.6\% of SCCs directly resulted in death [8].

Despite their low metastatic potential and distinctness from SCC, KAs are pathogenetically neoplastic. Ra et al. studied the molecular profiles of KAs in comparison to both regular skin and SCCs and found highly varied expression profiles; 1449 genes were expressed differently between the two diseases. This is compared to actinic keratosis, a purported pre-cursor to SCC, which only varied from SCCs by only 9 genes [9]. Likewise, other studies have supported molecular differentiation between the two in pathways ranging from growth to cellular maturation and apoptosis. Interestingly, many of the genetic derangements identified lead to increased apoptotic signaling, a potential cause of spontaneous involution.

The finding that skin tumors can be induced by exposure to a carcinogen in those with an oncogenic predisposition has been demonstrated [9]. HPV is one such potential carcinogen that has been associated with KAs. The potential for tattoo ink in this case to act as a carcinogen in KAs has not been proven but is theoretically within reason.

Tattoos have been known to cause a variety of dermatologic complications, albeit rarely. Most adverse reactions are infectious or allergic. Autoimmune reactions to various ink components are also described. One small trial estimated that $2.1 \%$ of tattoos develop infectious, allergic or granulomatous reactions [10]. However, the incidence of tumorous reactions within tattoos is so rare that it is not currently described. Less than 50 cases of skin cancer occurring within a tattoo, including melanoma, basal cell carcinoma, SCC and KAs have been reported. Of all reported cases, 28 were associated with red ink, 17 of these being KAs; only two of these cases described eruptive or multiple KAs arising solely within red ink portions of the tattoos [5]. Thus, while an association with red ink can be proposed, the tendency for eruptive KAs to occur within red ink is exceedingly rare.

This case appears to highlight much of this information. The patient appears to have had a reaction to the red ink used to re-ink his tattoo band. In concert with the findings of Ra, et al. it seems that this ink may have acted as a carcinogen. It is unknown what phenotypical characteristics make up a predisposed individual for KAs, though this patient's Fitzpatrick 1 skin type does predispose him to non-melanoma skin cancers such as basal cell carcinoma and SCC. The eruption is inconsistent with syndromal KAs based upon the localized nature of the lesions. Clinically, the lesions were perfectly consistent with typical KA le- 
sions. Interestingly, they also followed the pattern of spontaneous involution as the patients saw some improvement over the course of eight weeks without treatment. Acitretin therapy appears to have been successful in obtaining near clearance with successful eradication at one-year follow-up.

\section{Conflicts of Interest}

The authors declare no conflicts of interest regarding the publication of this paper.

\section{References}

[1] Sullivan, J.J. (1997) Keratoacanthoma: The Australian Experience. The Australasian Journal of Dermatology, 38, S36-S39. https://doi.org/10.1111/j.1440-0960.1997.tb01007.x

[2] Reizner, G.T., Chuang, T.Y., Elpern, D.J., Stone, J.L. and Farmer, E.R. (1993) Basal Cell Carcinoma and Keratoacanthoma in Hawaiians: An Incidence Report. Journal of the American Academy of Dermatology, 29, 780-782. https://doi.org/10.1016/S0190-9622(08)81701-X

[3] Ko, C.J. (2010) Keratoacanthoma: Facts and Controversies. Clinics in Dermatology, 28, 254-261. https://doi.org/10.1016/j.clindermatol.2009.06.010

[4] Ko, C.J., McNiff, J.M., Bosenberg, M. and Choate, K.A. (n.d) Keratoacanthoma: Clinical and Histopathologic Features of Regression. Journal of the American Academy of Dermatology, 67, 1008-1012. https://doi.org/10.1016/j.jaad.2012.02.041

[5] Kluger, N. (2010) Issues with Keratoacanthoma, Pseudoepitheliomatous Hyperplasia and Squamous Cell Carcinoma within Tattoos: A Clinical Point of View. Journal of Cutaneous Pathology, 37, 812-813. https://doi.org/10.1111/j.1600-0560.2009.01375.x

[6] Kwiek, B. and Schwartz, R.A. (2016) Review: Keratoacanthoma (KA): An Update and Review. Journal of the American Academy of Dermatology, 74, 1220-1233.

[7] Cavicchini, S., Tourlaki, A., Lunardon, L., Boneschi, V. and Gianotti, R. (2013) Amelanotic Melanoma Mimicking Keratoacanthoma: The Diagnostic Role of Dermoscopy. International Journal of Dermatology, 52, 1023-1024. https://doi.org/10.1111/j.1365-4632.2011.05025.x

[8] Savage, J.A. and Maize, J.C. (n.d) Keratoacanthoma Clinical Behavior: A Systematic Review. American Journal of Dermatopathology, 36, 422-429.

[9] Ra, S.H., Su, A., Li, X., Zhou, J., Cochran, A.J., Kulkarni, R.P. and Binder, S.W. (2015) Keratoacanthoma and Squamous Cell Carcinoma Are Distinct from a Molecular Perspective. Modern Pathology, 28, 799-806. https://doi.org/10.1038/modpathol.2015.5

[10] Kazandjieva, J. and Tsankov, N. (2007) Tattoos: Dermatological Complications. Clinics in Dermatology, 25, 375-382.

https://doi.org/10.1016/j.clindermatol.2007.05.012 